\title{
Aproximación a la representación social de los Jóvenes NiNi. Análisis desde la comunidad educativa
}

\section{Approach to the social representatio of Young NEETs. Analysis from the educational community}

\author{
ANA CRISTINA RUIZ MOSQUERA \\ Universidad de Málaga. Facultad de Estudios Sociales y del Trabajo \\ ORCID: 0000-0003-1920-5834 \\ MARÍA DE LAS OLAS PALMA GARCÍA \\ Universidad de Málaga. Facultad de Estudios Sociales y del Trabajo \\ ORCID: 0000-0003-1271-5604
}

Recibido: 02/02/2021

Aceptado: 27/07/2021

\begin{abstract}
Resumen: Se presenta un estudio descriptivo con el objetivo de aproximarnos a la representación social de los jóvenes NiNi, jóvenes que se encuentran sin estudiar ni trabajar. Hipótesis: la representación social creada describe a jóvenes pasivos, que no quieren estudiar ni trabajar. Utilizando metodología cualitativa, se realizaron entrevistas a 28 personas vinculadas al sistema educativo. Resultados: el término NiNi se ha incluido en nuestro vocabulario sin haber realizado un acercamiento a las circunstancias y características del colectivo. Conclusiones: existe una representación social concisa sobre estos jóvenes por parte de los distintos agentes que componen la comunidad educativa y la necesidad de profundizar en el conocimiento sobre este colectivo para el diseño de intervenciones que favorezcan la inclusión social de los jóvenes que atraviesen esa situación.
\end{abstract}

Palabras Clave: Representación social; jóvenes NiNi; comunidad educativa; exclusión social.

\begin{abstract}
A descriptive study is presented with the aim of approaching the social representation of young NEETs, young people who are neither studying nor working. Hypothesis: the social representation created describes passive young people, who do not want to study or work. Using qualitative methodology, interviews were conducted with 28 people linked to the educational system. Results: the term NiNi has been included in our vocabulary without having made an approach to the circumstances and characteristics of the group. Conclusions: there is a concise social representation of these young people by the different agents that make up the educational community and the need to deepen knowledge about this group for the design of interventions that favor the social inclusion of young people who go through this situation.
\end{abstract}

Keywords: Social representation; young NEETs; educative community; social exclusion. 


\section{INTRODUCCIÓN}

La Teoría de las Representaciones Sociales es una teoría en constante desarrollo y debate desde que surgiese en Francia en la década de 1960. En la actualidad, más de 50 años después de su primera formulación, el debate teórico, las investigaciones empíricas y la integración de planteamientos de orden multidisciplinar son indicadores de su evolución y actualidad. Se trata de una propuesta teórica que sugiere un planteamiento metodológico tan novedoso como interesante dentro del análisis del sentido común y de lo cotidiano, en nuestro caso, el reconocido por la sociedad española como fenómeno NEET (NiNi en su adaptación al castellano), por lo que se plantea como una explicación útil en el estudio de la construcción social de esta realidad.

La Representación Social nace tras la aparición de una situación nueva o bien de un fenómeno desconocido o de un evento inhabitual, como el que supone que existan jóvenes que no formen parte del sistema educativo ni del mercado laboral. Las representaciones sociales se caracterizan de manera más genérica como entidades operativas para el entendimiento, la comunicación y la actuación cotidiana. Se trata de conjuntos estructurados o imprecisos de nociones, creencias, imágenes, metáforas y actitudes con los que los actores definen las situaciones y llevan a cabo sus planes de acción (Jodelet, 1984).

En la actualidad no existe una definición exacta de NEET o joven que ni estudia ni trabaja. A nivel internacional la Organización de Naciones Unidas define este sector de la población como aquellas personas de edades entre 15 y 24 años que ni estudian ni trabajan, sin embargo, la Organización de Cooperación para el Desarrollo Económico describe a este sector de la población contemplando a los jóvenes de entre 15 y 29 años. Es por eso que se trata de un término más que de un concepto pues en el ámbito internacional no existe consenso sobre su definición. El contexto de España muestra que el sistema no es capaz de proveer suficiente oferta de empleo y formación a los jóvenes, de hecho, desde el segundo semestre del año 2009 los datos del impacto de la crisis económica entre los distintos colectivos juveniles, compuesto por la población de edades comprendidas entre los 16 y los 29 años, han sido cada vez más alarmantes (Observatorio de la Juventud en España, 2011) dando paso a la creación de una nueva categoría social, fenómeno NiNi, concepto que se ha conformado durante estos últimos años en nuestra sociedad, conocido y reconocido por todos los sectores sociales. Bajo dicho concepto se clasifican a personas que se encuentran en situación de desempleo, sin recibir forma- ción y sin ejercer ningún tipo de práctica. Navarrete (2011) describe esta compleja situación que engloba a jóvenes de todo tipo que presentan distintas circunstancias de actividad, formación, ocio, etc. identificados de manera inhabitualmente redundante como: "Jóvenes con trabajo", "con trabajo, pero a ver lo que dura...", "estudiando", "estudiando, pero después ¿qué va a hacer si no hay trabajo ni con estudios?

En cuanto a la visión social de esta situación, las personas se cuestionan ¿existe algo peor? Se afirma que se trata de un fenómeno de trayectorias heterogéneas (Torre y Baquerín, 2017) encerrando una connotación negativa caracterizada por la ausencia de atributos fundamentales en el proceso de socialización, como son la formación y el trabajo. Los jóvenes clasificables en España como "generación Y" están desarrollando su camino hacia la inserción laboral en condiciones aún más adversas que las afrontadas por la generación precedente ("generación X"), al coincidir la crisis económica experimentada a nivel nacional con su acceso a la edad laboral (Vallejo, 2017). Este lema se fue instalando poco a poco en la sociedad, sobre todo divulgándose en los medios de comunicación de masas como un correlato confirmativo del discurso pretendidamente lógico, racional, realista y definitivo que muestra así ante la opinión pública los efectos destructivos de la crisis (Observatorio de la juventud en España, 2011). De esta forma se ha establecido este sector de personas jóvenes que ni estudian, ni trabajan, ni se forman (NiNi) como un buen indicador del tamaño de la población en riesgo de exclusión social. Aunque el empleo en sí mismo tampoco es determinante para salir de la trampa de la pobreza (Andrade y Jiménez-Bandala, 2018) se puede establecer como indicador de inclusión social.

Explorar la representación social de este sector de la población permite aproximarse al análisis de la forma en que se describe, interpreta y se da sentido a este concepto. Según Abric (1994), el rol de las representaciones sociales se sitúa al mismo tiempo tanto al principio como al final de los juicios y de las conductas de los individuos y de los grupos con respecto a un objeto, a una situación o a un evento dado.

\section{LA REPRESENTACIÓN SOCIAL SOBRE LOS JÓVENES NINI}

Según Moscovici (1961) las representaciones sociales se crean a raíz de condiciones en que son pensadas y constituidas, teniendo como principal factor el hecho de surgir en momentos de crisis y de conflictos, es decir, momentos de cambio, como el que sufrió la sociedad española durante los años de crisis económica. Basado en las comprobaciones he- 
chas en su investigación, este autor deduce tres condiciones que provocan el nacimiento de estas representaciones sociales: la dispersión de la información, la focalización del sujeto individual y colectivo y la presión a la inferencia del objeto socialmente definido.

En primer lugar, la dispersión de la información viene expresada por la falta de suficiencia y desorganización de la información con la que la sociedad cuenta sobre un determinado hecho u objeto, es decir, los datos de que disponen la mayor parte de las personas para responder a una pregunta, para formar una idea o propósito de un objeto preciso, son generalmente limitados o bien demasiado abundantes (Moscovici, 1999). Tal como se ha señalado anteriormente, desde mediados de la crisis económica la información sobre los jóvenes NiNi ha sido tan abundante como diversa, cumpliendo la condición de dispersión de la información que Moscovici propone.

En segundo lugar, la focalización se define en palabras de investigadores como Banchs (1984) y Herzlich (1979) en términos de implicación o atractivo social de acuerdo con los intereses particulares que se mueven dentro del individuo perteneciente a los grupos sociales, de ello se deduce que la focalización será diversa. Según Moscovici (1961), una persona o una colectividad se focalizan porque están involucradas en la interacción social como hechos que alteran las ideas y opiniones.

El tercer factor viene definido por la presión que ejerce la sociedad reclamando opiniones, posturas y acciones acerca de los hechos que suscitan interés público, esto es conocido como presión a la inferencia. En el desarrollo del día a día de las personas, las circunstancias y las relaciones que se establecen entre estas exigen del grupo social o de sus componentes, capacidad de responder en todo momento. Por otra parte, para Banchs (1984), las exigencias grupales para el conocimiento de determinada situación, evento u objeto van incrementando conforme su relevancia aumente. Por lo tanto, el fin último sería encontrarse incluido en las conversaciones, poder inferir y generar opiniones de forma rápida, ofreciendo un discurso más o menos elaborado.

La confluencia de estos tres factores permite la aparición del proceso de formación de una representación social, iniciándose dicho proceso desde determinados grupos sociales con influencia en los fenómenos de los que se trate. En relación a los jóvenes NiNi, las personas relacionadas con el sistema educativo de forma directa, disponen a priori de un sistema de comprensión y de evaluación concreto sobre ellos. Este sistema está regido por el pensamiento social caracterizado por una lógica relacional y no racional como apuntan Rateau y Lo Monaco (2013). Es por eso que cuando se define el concepto de joven que ni estudia ni trabaja dentro del contexto edu- cativo, aparecen distorsiones específicas de la población y del sentido que se le atribuye a este fenómeno social, quienes participan en una comunidad comparten patrones de actividad y de pensamiento. Se produce distinción entre el sujeto que se considera ideal y el sujeto social (Rouquette, 1994) al mismo tiempo que como indica Guillemi (1999) el pensamiento racional diverge de forma significativa del pensamiento social.

El estudio de la representación social de los jóvenes NiNi permite reconocer los modos y procesos de constitución del pensamiento social, a través del discurso y la práctica que en torno a este fenómeno se genera (Abric, 1994). De esta forma, es posible aproximarse a la "visión de mundo" que las personas o grupos tienen sobre estos jóvenes, pues el conocimiento del sentido común es el que las personas utilizan para actuar o tomar posición ante los distintos objetos sociales (Araya, 2002). Acercarse a este conocimiento, construido entre las personas que forman parte de la comunidad educativa es imprescindible para la comprensión de la realidad objeto de este trabajo, ya que los agentes presentes en dicha comunidad son claves en la relación joven-formación. Por otro lado, la novedad del fenómeno NEET también anima a explorar este conocimiento, al disponer aún de información limitada e incompleta para los grupos sociales que se ven implicados en la emergencia de este sector en la sociedad. En coherencia con este planteamiento, la finalidad del presente estudio es explorar cuál es la representación social del fenómeno NiNi, concretamente en un sector de la población directamente relacionado con la naturaleza de su situación: La comunidad educativa.

\section{MÉTODO}

\section{Tipo de estudio}

El presente estudio se realiza bajo un diseño de tipo descriptivo y transversal, ya que su objetivo es recolectar datos en un momento concreto, en un tiempo único (Hernández, Fernández y Baptista, 2006) que nos permita establecer una representación social de los jóvenes NiNi en la sociedad a partir de agentes representativos del sistema educativo, utilizando el método deductivo para el análisis de contenido, obteniendo una serie de conclusiones a partir de premisas generales.

\section{Participantes}

Este estudio se ha dirigido a personas relacionadas con el sistema educativo, es decir, que formaban parte de la comunidad educativa. 
La muestra de participantes estuvo compuesta por un conjunto de personas relacionadas con la acción educativa, cuyo desempeño diario persigue metas relacionadas con el desarrollo educativo y formativo tanto individual como cultural de los jóvenes. Así entendida, la comunidad educativa se configura como un sistema abierto que se alimenta y desarrolla a partir de elementos de diferentes ámbitos -social, político, cultural, ideológico, económico-y sus correspondientes interacciones. Al mismo tiempo esta comunidad está compuesta por un conjunto de subsistemas que se relacionan, interactúan y que presentan una serie de objetivos comunes, entre los que destaca el éxito educativo de los jóvenes.

Desde la perspectiva de Bronfenbrenner (1987), la familia y la escuela son microsistemas en los que los menores se desarrollan, mientras que la comunidad educativa sería el mesosistema en el que se interrelacionan ambos microsistemas. El reconocimiento de estas interacciones resulta básico para comprender los patrones de actividad, los roles y, en general, el tránsito de los menores a la vida adulta.

Concretamente, la población de estudio se conformó inicialmente por 50 participantes ( 25 de ambos sexos) vinculados al sistema educativo por estar trabajando en la escuela (profesorado, equipo directivo, profesionales del Equipo de Intervención Psicosocial) por haber pertenecido a la escuela (ex-alumnado) y familiares (padres y madres de ex-alumnado) y que mantienen contacto directo con jóvenes que ni estudian ni trabajan. Para la elección de participantes se utilizó la técnica de conglomerado que permitió reducir el número de entrevistas para ofrecer resultados más precisos, resultando una muestra final compuesta por 28 sujetos, cuyas edades oscilan entre 27 y 63 años y presentan los siguientes rasgos sociodemográficos:

TABla I. Perfil SOCIODEMOgRÁfico de PARTICIPANTES

\begin{tabular}{|c|c|c|c|c|}
\hline Sujeto & $\begin{array}{c}\text { Año de } \\
\text { nacimiento }\end{array}$ & Sexo & Profesión & Hijos/as \\
\hline E1 & 1966 & Mujer & Profesora & $\mathrm{SI}$ \\
\hline E2 & 1958 & Hombre & Profesor & $\mathrm{SI}$ \\
\hline E3 & 1970 & Mujer & Profesora & $\mathrm{SI}$ \\
\hline E4 & 1973 & Hombre & Profesor & $\mathrm{SI}$ \\
\hline E5 & 1962 & Hombre & Economista & $\mathrm{SI}$ \\
\hline E6 & 1972 & Hombre & Profesor & $\mathrm{SI}$ \\
\hline E7 & 1973 & Mujer & $\begin{array}{l}\text { Analista de Ries- } \\
\text { gos }\end{array}$ & $\mathrm{SI}$ \\
\hline E8 & 1965 & Mujer & Profesora & $\mathrm{SI}$ \\
\hline E9 & 1994 & Hombre & Estudiante & NO \\
\hline E10 & 1974 & Mujer & Logopeda & $\mathrm{SI}$ \\
\hline E11 & 1955 & Hombre & Economista & $\mathrm{SI}$ \\
\hline E12 & 1975 & Hombre & Administrativo & NO \\
\hline
\end{tabular}

\begin{tabular}{|lllll|}
\hline E13 & 1971 & Mujer & Autónoma & SI \\
\hline E14 & 1981 & Mujer & Profesora & NO \\
\hline E15 & 1989 & Mujer & $\begin{array}{l}\text { Asistente de Ge- } \\
\text { rencia }\end{array}$ & NO \\
\hline E16 & 1959 & Mujer & $\begin{array}{l}\text { Jefa de Estudios y } \\
\text { profesora }\end{array}$ & SI \\
\hline E17 & 1969 & Mujer & Economista & SI \\
\hline E18 & 1974 & Hombre & Profesor & SI \\
\hline E19 & 1992 & Mujer & Becaria & NO \\
\hline E20 & 1975 & Mujer & Profesora & SI \\
\hline E21 & 1989 & Hombre & Auditor & SI \\
\hline E22 & 1986 & Hombre & Becario & NO \\
\hline E23 & 1956 & Hombre & Economista & SI \\
\hline E24 & 1948 & Hombre & Jubilado & SI \\
\hline E25 & 1990 & Hombre & Estudiante & NO \\
\hline E26 & 1957 & Hombre & Administrativo & SI \\
\hline E27 & 1949 & Mujer & Jubilada & NO \\
\hline E28 & 1967 & Hombre & Administrativo & NO \\
\hline
\end{tabular}

Para el análisis anonimizado de los resultados, cada participante se identifica con un código número tal como se recoge en la tabla anterior.

\section{Instrumento}

Se utiliza la entrevista como técnica de recogida de información. Ha sido diseñada de forma específica para el presente estudio, compuesta por 12 ítems de carácter abierto mediante los que se ha obtenido información relativa al fenómeno NiNi. Con el objetivo de poder realizar comparaciones y posibles contrastes entre las entrevistas que permita organizar conceptualmente los datos y presentar la información siguiendo cierta regularidad, se han establecido once categorías para su análisis de contenido, atendiendo a los elementos que Moscovici (1979, p.45) plantea como componentes de la representación social: la información, el campo de representación (imagen y opiniones) y las actitudes.

\section{Procedimiento}

Se informó a los participantes de la finalidad del estudio y se estableció con cada uno de ellos un compromiso de participación y un documento de confidencialidad, la firma de estos documentos fue previo a la cita para la entrevista. Las entrevistas se realizaron de forma individual a lo largo del 2018, con una duración de entre 30 y 45 minutos. Una vez analizadas auditivamente, se pasó a la transcripción de las 28 entrevistas seleccionadas para formar parte de la muestra. 
El tratamiento de los datos se ha llevado a cabo mediante el análisis de contenido deductivo. Se han eliminado cualquier tipo de referencias a datos personales estableciendo en el proceso garantía de confidencialidad y principios éticos. Tras su lectura inicial, se ha realizado el análisis de contenido, identificando la frecuencia de aparición de las categorías y llevando a cabo su posterior tratamiento cualitativo, obteniéndose los siguientes resultados.

\section{RESULTADOS}

Los resultados se muestran agrupados según las dimensiones previstas para su análisis: Información, Campo de representación y Actitudes.

\section{Información en torno a los jóvenes $\mathrm{NiNi}$}

Esta dimensión incluye la suma de conocimientos con que cuenta el grupo de participantes sobre el fenómeno NiNi. Para analizar esta dimensión contamos con las siguientes subcategorías: concepto de NiNi, nivel formativo o de experiencia profesional y el conocimiento sobre aquellas políticas públicas dirigidas a jóvenes NiNi. A continuación, se muestran los resultados obtenidos según dichas subcategorías.

\section{Concepto NiNi}

En cuanto al concepto de joven $\mathrm{NiNi}$, la totalidad de participantes definen este fenómeno como aquellos adolescentes o jóvenes que en la actualidad no está estudiando ni trabajando $(n=28)$.

"Con el término NiNi se identifican los jóvenes de entre 19 y 29 que por decisión propia ni estudian ni trabajan o bien que han finalizado sus estudios y no encuentran trabajo, no esforzándose por seguir formándose o buscar trabajo" (E11)

"Jóvenes desempleados y sin ningún nivel de estudios. La mayoría pertenecen a clases sociales desfavorecidas económicamente" (E3).

Para la mayoría de los entrevistados $(n=23)$ son además jóvenes que no han superado la Educación Secundaria Obligatoria, "no terminan los estudios básicos y esperan a cumplir la edad para irse del colegio" (E22)

"Han terminado a duras penas la ESO o se han quedado a las puertas de terminar los estudios obligatorios y no están trabajando ni tienen perspectivas a corto plazo de trabajar" (E6).

$Y$ que continúan conviviendo con sus padres $(n=25)$, al definirlos como "jóvenes entre 16 y 30 años que viven con sus padres y sin preocupaciones por su futuro profesional" (E2).
Por último, se incorpora al concepto construido por los participantes la falta de interés e inquietudes de cara al futuro $(n=20)$. Se consideran "jóvenes que ni estudian ni trabajan, y que suelen cumplir un perfil de chicos despreocupados por su futuro, sin motivaciones ni objetivos que cumplir más allá que el de divertirse" (E19) que "no muestran interés en estudiar ni en encontrar un empleo" (E7).

Nivel formativo de los jóvenes NiNi y experiencia profesional Los resultados muestran unanimidad a la hora de referirse a la cualificación y experiencia de los jóvenes que ni estudian ni trabajan siendo ambas bajas, casi inexistente $(\mathrm{n}=28)$. "Los NiNi de mi entorno no tienen ni formación ni experiencia laboral" (E4).

"Ninguna experiencia profesional y un nivel formativo aceptable pero poco competitivo" (E17).

"En general el nivel formativo es bajo y escasa o nula experiencia profesional" (E23).

\section{Políticas Públicas dirigidas a los jóvenes NiNi}

En cuanto al conocimiento y desarrollo de las políticas públicas que se están llevando a cabo tanto a nivel local, autonómico como nacional en atención a estos jóvenes, las personas entrevistadas parten inicialmente de un elevado grado de desconocimiento $(n=23)$, considerando que existe falta de difusión de dichas políticas $(n=26)$ y de efectividad $(n=24)$. Muestra de ello son los siguientes relatos: "Sinceramente no las conozco, por lo que la difusión entre los ciudadanos es insuficiente, y por tanto, también para el sector de jóvenes afectados" (E19).

"No conozco las políticas que se están llevando a cabo con esta población" (E1).

"No las conozco, pero no deben ser eficaces porque cada vez veo más jóvenes sin hacer nada" (E12).

Los participantes centran la efectividad de estas políticas en aquellas que pudieran ir dirigidas al fomento de la educación y la prevención del abandono escolar temprano, tal como refleja el siguiente extracto: "entiendo que las medidas se deben tomar desde el ámbito educativo en los centros escolares y también en el ámbito familiar, con ayuda a los padres y madres para saber afrontar este problema» (E6).

Por otro lado, quienes sí las conocen valoran que su alcance no llega a todo el mundo: "Conozco las políticas públicas y las considero ineficaces porque muchas veces para determinados cursos de formación de empleo se requiere una formación que estos jóvenes en su mayoría no tienen. No llegan a gran parte de la sociedad" (E2).

"Es verdad que existen politicas de prevención de NiNi pero no siempre llegan a toda la población" (E9). 


\section{Campo de representación (imagen y opiniones) sobre los jóvenes Nini}

Los resultados obtenidos ponen de manifiesto la organización del contenido de la representación sobre los jóvenes Nini de forma jerarquizada, existiendo por lo tanto una amplia variedad dentro del grupo de participantes. Esta categoría expresa el carácter del contenido, las propiedades cualitativas e imaginativas de la representación social. Se trata de un campo que integra las informaciones analizadas anteriormente en un nuevo nivel organizativo respecto a sus fuentes directas o inmediatas. Las subcategorías que componen el campo de representación en nuestra investigación son las siguientes: rutina de los NiNi, consecuencias para su salud, motivación para la superación de esta situación, influencia de la formación de padres y madres de jóvenes NiNi y consecuencias para la sociedad.

\section{El día a día de los NiNi}

En cuanto a la imagen y opiniones que suscita la rutina de los NiNi tras el análisis del discurso de las entrevistas realizadas las palabras más mencionadas han sido redes sociales $(\mathrm{n}=$ $26)$, dormir $(n=24)$, videojuegos $(n=24)$, salir con los amigos ( $n=23)$ y móvil $(n=22)$. Según se observa en los siguientes extractos:

"Lo que hacen es dormir mucho, comer, van de compras, se cuidan físicamente, están con amigos o conectados a redes sociales" (E10).

"Pasan el día en sus casas dedicando mucho tiempo a jugar a videojuegos, a ver la tele, a conectarse a las redes sociales, pendientes del móvil y salen con sus amigos" (E7).

A su vez se hace referencia al tiempo que pasan en casa, que sigue siendo la de sus padres $(n=22)$, a la relación que mantienen con jóvenes de perfil similar $(n=19)$ y a la falta de objetivos que presentan en sus vidas $(n=16)$.

"Este tipo de jóvenes pasan el día a día durmiendo, alimentándose en casa de sus padres y relacionándose con sus amigos" (E1).

"Su día a día es monótono y repetitivo. Son perfiles que siguen viviendo en casa de sus padres, se levantan tarde, se acuestan muy tarde, usan mucho el móvil para comunicarse con sus amigos vía "whatsapp", usan redes sociales y videojuegos para gastar su tiempo y salen a dar una vuelta con sus amigos" (E6).

"Algunos ninis están acostumbrados al confort que sus padres les han dado durante su niñez y adolescencia, por tanto, no se esfuerzan por salir adelante; se entretienen con la $T V$, videoconsolas e Internet. Otros, con muy pobres ex- pectativas para el futuro, hacen mandados o cuidan la casa durante el día. La culpa la tienen sus propios progenitores por no haberles inculcado la necesidad de valerse por sí mismos" (E15).

\section{Consecuencias en la salud de estos jóvenes}

Según los participantes las consecuencias que tiene estar sin estudiar ni trabajar para estos jóvenes son la tendencia a la depresión $(n=26)$, el establecimiento de hábitos no saludables [alcohol y drogas] $(n=24)$, el sedentarismo $(n=21)$ y el desarrollo de episodios de ansiedad ( $n=18)$.

"Su salud se ve resentida tanto física como emocionalmente pasando por depresiones" (E28).

"Influye sobre todo en su salud mental y emocional. Sienten una fuerte frustración, apatía, confusión e incluso, sentimientos de desesperanza" (E7).

"Esta situación obviamente influye en su salud. Por un lado, muchas enfermedades están muy relacionadas con la frustración de verse fuera de la sociedad, y por otra está el riesgo a caer en hábitos nocivos para la salud" (E21).

\section{Motivación para la superación de esta situación}

Tal como se observa en los siguientes relatos los resultados muestran la percepción de jóvenes faltos de motivación $(n=26)$ y desanimados $(n=22)$ ante la situación que están viviendo: "Son personas que no encuentran la motivación necesaria para tratar de esforzarse y mejorar su situación, obteniendo una cualificación para conseguir un trabajo que les permita superar esa fase de desencanto con la vida" (E5).

"No son conformistas pero su día a día les hace vivir desmotivados" (E27).

"Son personas que no tienen motivación y están estancados en un tipo de vida que no les permite desarrollarse" (E9).

Se consideran que son jóvenes con sus necesidades básicas cubiertas $(n=17)$ y que se conforman con el estilo de vida que tienen $(n=17)$.

"Por distintas circunstancias que les ha tocado vivir: (ambiente familiar, situación económica, relaciones sociales, etc, se han acostumbrado a vivir así y ya les resulta cómodo y normal. Si intentaran ponerse alguna meta que les motivase y le resultara atractiva, aunque fuera algo muy básico y vieran que son capaces de conseguirla, los ayudaría a salir de ahi" (E3).

"Viven muy bien en casa de sus padres sin muchas exigencias y demasiadas comodidades, no les preocupa nada más, viven el momento sin ningún interés por el futuro" (E22). "Síntomas comunes podrían ser la normalización de la situación de "no hacer nada" y la falta de motivación para 
revertir esta situación. Además, otra característica que bajo mi punto de vista pueden tener estas personas son un entorno de iguales permisivos y con el mismo problema, esto hace que aún la normalización de la situación sea mayor" (E21).

Influencia de la formación de los padres y madres en jóvenes que ni estudian ni trabajan

El $100 \%$ de los entrevistados $(n=28)$ hace referencia en su discurso a la importancia de la educación, formación y experiencia de la sociedad en general, no estableciéndose una relación directa entre padres sin formación o experiencia e hijos que ni estudien ni trabajen. Los participantes $(n=19)$ indican la importancia de la transmisión de valores como el esfuerzo, el compromiso y el valor de trabajo de padres a hijos, tal como se evidencia en los siguientes relatos: "es muy importante la formación y experiencia que tengan todas las personas, aunque creo que no es indicativo para que sus hijos sean NINI; es decir, padres con estudios universitarios pueden tener hijos NINI y sin embargo, padres sin estudios no necesariamente tendrán hijos NINI" (E1).

"Además de la formación y experiencia de los padres, éstos tienen que inculcar a sus hijos desde pequeños una disciplina del estudio y un sentido de la responsabilidad, premiándolos cuando sus resultados académicos sean buenos haciéndoles sentir que se sienten orgullosos de ellos. Los padres tienen que ejercer su autoridad de forma responsable $y$ establecer límites" (E7).

"Creo que cuanta mayor sea la formación de los padres, menos posibilidades de que los hijos sean NiNi hay, si bien no existe garantía alguna de que la formación educativa de los padres garantice que los hijos la obtengan. Todo va a depender de la educación que los padres les den a sus hijos" (E8).

\section{Consecuencias para la sociedad}

Los participantes trasladan preocupación por la situación actual de los jóvenes Nini $(n=26)$, que consideran una pérdida de potencial $(n=8)$, que genera incertidumbre sobre futuras generaciones $(n=23)$, aumento del paro juvenil $(n=19)$ e incapacidad de desarrollo por parte de estos jóvenes $(n=22)$.

"Muchas familias están soportando la situación, creándoles sensaciones de malestar por la incertidumbre del futuro social de estos jóvenes" (E2).

"Este fenómeno está afectando negativamente, porque nuestra sociedad se está empobreciendo cultural y económicamente. Ya que si no tenemos jóvenes que se formen adecuadamente tampoco podrán trabajar ni crear puestos de trabajos, ni cotizar y todo ello influye negativamente en el desarrollo de nuestro país" (E3).
"En la actualidad el fenómeno NINI está más olvidado, lo que no quiere decir que se haya solucionado, pero con esta situación se motiva un desequilibrio en la estabilidad del país y una desconfianza en los jóvenes, pues el relevo generacional peligra y se complica el crecimiento del país" (E19).

\section{Actitudes sociales hacia los jóvenes Nini}

Esta dimensión contiene la orientación favorable o desfavorable en relación a los jóvenes NiNi. Generalmente, para la aproximación a la representación social de cualquier fenómeno esta dimensión es la más estudiada ya que incluye un componente comportamental y de motivación hacia ello. En nuestro estudio su análisis ha aportado los siguientes resultados.

\section{Relación con la exclusión social}

La situación de no estudiar ni trabajar sitúa a estos jóvenes fuera de la sociedad ( $n=19)$, "cada vez hay más jóvenes sin estudio ni trabajo y con muchas dificultades a la hora de conseguir y mantener un trabajo, eso les aparta de la sociedad" (E14).

También se hace mención al término de autoexclusión $(n=6)$, "se podría decir que es un proceso de autoexclusión, aunque bien es cierto que a los que pretenden integrarse les puede resultar muy difícil ante la falta de oportunidades que les permitan acceder a los beneficios que la sociedad ofrece" (E5).

"No pienso que lleguen al límite de marginados sociales, pues creo que en la mayoría de los casos son situaciones voIuntarias que se podrían evitar si estos NINI quisieran buscar solución" (E19).

\section{Connotación negativa del término}

En cuanto a la connotación del término, el 100\% de los entrevistados hacen referencia a que se trata de una característica negativa en cuanto a reconocimiento social. Las palabras que más se identifican con el reconocimiento social que se le da son las de vagos ( $n=14)$ y "parásitos sociales" $(n=13)$, tal como muestran los siguientes relatos: "Ser nini es percibido como totalmente negativo en nuestra sociedad" (E18).

"La sociedad los tachan de vagos y personas poco recomendables que viven del esfuerzo de su entorno familiar" (E2).

"La sociedad es crítica con estos jóvenes y los ven como un problema en el futuro desarrollo del país" (E19).

"La sociedad los identifica como flojos" (E20).

"Los NiNi no están bien vistos, son considerados como una especie de "parásitos" de la sociedad" (E3). 


\section{Calidad de vida}

El total de entrevistados relacionan el término calidad de vida con haber superado estudios y tener un trabajo que permita al individuo desarrollar todo su potencial. En general, hacen referencia a que los jóvenes NiNi no gozan de calidad de vida $(n=23)$ debido a que no tienen trabajo $(n=23)$, no pueden disfrutar de todos los aspectos de la vida $(n=12)$ y esto les provoca problemas de salud mental y frustración $(n=9)$.

"Pienso que los NiNi no tienen calidad de vida porque están fuera del ámbito de estudiantil y del laboral, que te proporciona el conocer gente, relacionarte, tener una obligación de asistir a clase, al trabajo, conocer, diferentes puntos de vistas" (E22).

"No tienen calidad de vida porque este hecho está retrasando su desarrollo en todos los sentidos: no se pueden independizar, no pueden formar una familia, no pueden hacer planes de futuro" (E27).

\section{DISCUSIÓN}

El concepto europeo de joven NiNi según la Organización Internacional del Trabajo (2015) hace referencia a "jóvenes que no trabajan, ni estudian ni está en práctica, personas entre los 15 y los 24 años que están en situación de desempleo y fuera del sistema educativo". Más concretamente, en España, según la Fundación Europea para la mejora de las condiciones de vida y de trabajo (2011), el perfil de NiNi viene definido por jóvenes que, aunque no tengan empleo y no estén siguiendo educación o aprendizaje, gozan de un buen nivel formativo y de experiencia profesional. Sin embargo, la dimensión de información de la representación social de este sector de la población refleja un perfil de adolescente o joven, que efectivamente ni estudia ni trabaja, que no han superado los estudios de educación obligatoria y que, además, carecen de inquietudes de cara al futuro.

La representación social del joven NiNi obtenida en la presente investigación viene dada por niveles de estudios bajos o inexistentes, lo que se aleja de la definición propuesta por la Fundación Europea para la mejora de las condiciones de vida, la cual refleja jóvenes con buen nivel formativo. Esta dimensión está relacionada con las actitudes reflejadas a través de la connotación negativa que encierra este término (jóvenes definidos como vagos o parásitos sociales), fundamentadas en las definiciones sobre su rutina en la que emerge el paso del tiempo en redes sociales, salidas con el grupo de iguales, es decir, días carentes de objetivos. Además, la representación social refleja un perfil de adolescentes y jóve- nes faltos de motivación, desanimados, con sus necesidades básicas cubiertas y conformistas, sin intención de cambio de situación. Esto repercute de forma directa en su salud, tanto mental (trastornos depresivos, generación de adicciones y episodios de ansiedad) y con su salud física (sedentarismo como principal problema mencionado). También se refleja en la valoración que hacen sobre su calidad de vida. Para nuestra sociedad el tener un trabajo que desempeñar es considerado la acción que te permite disfrutar de todos los aspectos de la vida. Es por eso que la carencia de una ocupación (estudios o trabajo) se considera como una característica que no permite gozar a estos jóvenes de calidad de vida.

En cuanto a la categoría referente a la formación y experiencia profesional de los padres, se consideran factores de riesgo que los progenitores hayan estado desempleados (teniendo un $17 \%$ más de probabilidad) y que tengan bajo nivel de estudios, teniendo el doble de probabilidad de ser NiNi (Fundación Europea para la mejora de las condiciones de vida y de trabajo, 2011:4). Definición que corresponde a la aplicada a su representación social ya que todos los participantes refieren como esencial la educación, formación y experiencia de la sociedad en general, y en particular la importancia de la transmisión del valor de la educación. Se destaca la importancia de inculcar valores como el esfuerzo, el compromiso y el valor de trabajo de generación en generación.

El concepto de NiNi se ha configurado desde un primer momento como un joven excluido y marginado social (Morales, 2015) cuando lo correcto es entender esta situación como una característica que predispone a la exclusión social. Esta configuración se identifica en su representación social, la situación de no estudiar ni trabajar sitúa a los adolescentes y jóvenes fuera de la sociedad, aunque también se refleja la autodeterminación, cierto matiz de elección, ya que también se hace mención al término autoexclusión social por parte de este sector de la población. La representación social denota una sociedad que se preocupa por esta situación debido a la gran pérdida de potencial que esto supone. Además, se hace mención la incertidumbre por el futuro de la sociedad debido a la incapacidad de desarrollo por parte de las nuevas generaciones.

Debemos destacar la gran laguna de conocimiento que se pone de manifiesto cuando de políticas públicas dirigidas a estos adolescentes y jóvenes hablamos. Se trata de un dato tan significativo como preocupante, ya que su desconocimiento impide la orientación por parte de quienes están directamente relacionados con estos jóvenes NEET (familia, personal de centros educativos y grupo de iguales). Se pone de manifiesto la falta de difusión de estas políticas y la falta 
de efectividad debido a que no llegan a la población afectada. El tener información otorga poder y en este caso, la orientación directa por parte de familiares, profesores o amigos es fundamental para conseguir que estos jóvenes continúen formándose a través del sistema educativo o formen parte del mercado laboral para favorecer así su inclusión en la sociedad.

\section{CONCLUSIONES}

Pese a la heterogeneidad que caracteriza a los jóvenes que atraviesan la situación de NEET, de los resultados de nuestra investigación se pone de manifiesto una representación social clara y concisa sobre estos jóvenes por parte de los distintos agentes que componen la comunidad educativa: padres y madres, exalumnos y exalumnas y profesorado, es decir, personas relacionadas directamente con este sector de la población. Confirmando la hipótesis de la que partíamos, el término joven NiNi encierra una gran connotación negativa que se pone de manifiesto en las distintas dimensiones analizadas que conforman la representación social de este sector de la población. El término NiNi ha entrado en el vocabulario de las políticas públicas en general y de nuestra sociedad en particular sin que se haya estudiado lo que realmente es y lo que pretende reflejar. En él se engloban jóvenes en situación de vulnerabilidad, desvinculados de las vías de la inclusión social: la educación, la formación y el mercado laboral. Jóvenes dependientes de sus familiares, con tendencia al desarrollo de problemas de salud mental debido a la falta de actividad y a su desconexión de la sociedad.

El colectivo de los Jóvenes NEET reclama una actuación preventiva y no paliativa, reconocer el problema de que existan adolescentes y jóvenes fuera de los circuitos de inclusión social es una tarea necesaria pero insuficiente cuando del diseño de políticas públicas estamos hablando. Las herramientas elegidas para solucionarlo están fracasando desde sus orígenes debido a su falta de difusión y accesibilidad por parte tanto de los adolescentes y jóvenes que se ven afectados por esta situación como de su entorno más inmediato, cuya orientación consideramos especialmente esencial en la prevención del fenómeno NEET. Proponemos repensar esas herramientas para abordar los problemas desde antes de que los jóvenes se desvinculen del sistema educativo sin que tengan posibilidades de insertarse en el mercado laboral. No obstante, nuestro estudio pone de manifiesto la necesidad no sólo de una educación sino de una educación y formación correcta ya que finalmente ambas variables son cara de una misma causa de empleo y desempleo. Se hace necesaria una orientación desde la adolescencia para favorecer la continuidad en el sistema educativo con el objetivo de recibir una formación correcta acorde a las características de cada individuo y a las necesidades del mercado laboral.

\section{REFERENCIAS BIBLIOGRÁFICAS}

Abric, J. C. (1994). Les représentations sociales: aspects théoriques. En J.C. Abric (Ed.), Pratiques sociales et représentations, 11-35. Paris: Presses universitaires de France.

Andrade, L. A., Jiménez-Bandala, C. A (2018). El desempleo y la probabilidad de caer en trampas de pobreza: consideraciones para países en vías de desarrollo. Revista Española de Investigaciones Sociológicas, 164, 3-20. http:// dx.doi.org/10.5477/cis/ reis. 164.3

Araya, S. (2002). Las representaciones sociales: Ejes teóricos para su discusión. Cuadernos de Ciencias Sociales, 127, 1-84.

Banchs, M. (1984). Las representaciones sociales: sugerencias sobre una alternativa teórica y un rol posible para los psicólogos sociales en Latinoamérica. En: Jiménez, B. (compilador) Aportes críticos a la Psicología social en Latinoamérica. Guadalajara: EDUC.

Fondo de Población de las Naciones Unidas (2014). Estado de la población mundial 2014. UNFPA:2014

Guillemi, C. (1999). La pensée sociale. Paris: Presses Universitaires de France.

Hernández, R; Fernández, C. y Baptista, P. (2006). Metodología de la investigación (4a ed.). DF, México: McGraw Hill.

Herzlich, C. L. (1979). La representación social: sentido del concepto. En Moscovici, S. (compilador). Introducción a la Psicología social. Barcelona: Planeta.

Jodelet, D. (1984). La representación social: fenómeno, concepto y teoría. En Moscovici, S. (compilador). Psicología social II. España: Paidós.

Morales, J. M. (2015). La respuesta comunitaria y española a la realidad de los ninis: los grandes damnificados por la crisis, 37. Murcia: Laborum, D.L.

Moscovici, S. (1961). El psicoanálisis, su imagen y su público. Buenos Aires: Huemul.

Navarrete, L. (dir.) (2011). Desmontando a ni-ni. Un estereotipo juvenil en tiempos de crisis. Madrid: Instituto de la Juventud.

Organización para la Cooperación y el Desarrollo Económico (2014). Panorama de la Educación Indicadores de la OCDE. OECD Publishing.

Organización Internacional del Trabajo (2015). Tendencias mundiales del empleo juvenil. Promover la inversión en 
empleos decentes para los jóvenes. Ginebra: Oficina Internacional de Trabajo.

Rateau, P., y Lo Monaco, G. (2013). La Teoría de las Representaciones Sociales: Orientaciones conceptuales, campos de aplicaciones y método. CES Psicología, 6(1), 22-42.

Rouquette, M. L. (1994). Sur la connaissance des masses. Essai de psychologie politique. Grenoble: Presses Universitaires de Grenoble.
Torre, L. y Baquerín, M. (2017). Los jóvenes argentinos que no estudian ni trabajan: déficit de integración social. Revista Española de Investigaciones Sociológicas, 158, $97-$ 116. http://dx.doi.org/10.5477/cis/reis.158.97

Vallejo, A. (2017). La generación $Y$ ante el desafío de su inserción laboral: realidades frente a estereotipos. Arbor, 193 (783): a375. http://dx.doi.org/10.3989/arbor.2017.783n1006 